\title{
Structural relaxation and self-diffusion in covalent amorphous solids: Silicon nitride as a model system
}

\author{
H. Schmidt ${ }^{\text {a),b) }}$ and W. Gruber \\ Institute of Metallurgy, Materials Physics Group, Clausthal University of Technology, \\ Robert-Koch-Strasse 42, D-38678 Clausthal-Zellerfeld, Germany \\ T. Gutberlet, M. Ay, and J. Stahn \\ Laboratory for Neutron Scattering, ETH Zürich \& Paul Scherrer Institut, CH-5232 Villigen PSI, \\ Switzerland \\ U. Geckle and M. Bruns \\ Institute for Materials Research III, Karlsruhe Research Center GmbH, Hermann-von-Helmholtz-Platz 1, D- \\ 76344 Eggenstein-Leopoldshafen, Germany
}

(Received 25 May 2007; accepted 4 July 2007; published online 23 August 2007)

\begin{abstract}
Neutron reflectometry and isotope multilayers were used to investigate self-diffusion in covalent amorphous solids during isothermal annealing and its correlation to structural relaxation. Amorphous silicon nitride was chosen as a model system. Neutron reflectometry is a superior method to measure very low self-diffusivities, occurring in covalent solids, by applying only short time anneals. This allows one to determine time dependent changes of diffusivity over a broad temperature range before crystallization of the metastable solid occurs. The measured nitrogen diffusivities decrease by more than one order of magnitude during annealing between 950 and $1150{ }^{\circ} \mathrm{C}$, while at the same time also a decrease of the atomic density is observed. We interpret this behavior as a structural relaxation of the amorphous network structure that is governed by annihilation of interstitial-like defects. () 2007 American Institute of Physics.
\end{abstract}

[DOI: $10.1063 / 1.2770821]$

\section{INTRODUCTION}

Structural relaxation in amorphous or glassy solids can be described as a rearrangement process of short-range disorder during annealing at elevated temperatures, which is generally irreversible. The solid transforms from a less stable to a more stable, but still metastable amorphous state. During the fabrication of amorphous materials by rapid cooling of a liquid or by deposition from the gas phase, a frozen-in highly distorted and strained structure is formed. Annealing may lead to a rearrangement of bond lengths, bond angles, a reduction of strain, and a redistribution of defects. ${ }^{1}$ A number of physical properties (vibrational, electronic, magnetic, mechanical, and thermodynamic parameters) may be altered during this process. As a consequence, parameters of critical technological importance such as the bulk modulus, the electrical resistivity, and the permeability become time dependent.

Structural relaxation is well known and observed in most types of amorphous materials such as silicate glasses, ${ }^{2}$ polymer glasses, ${ }^{3}$ metallic amorphous alloys, ${ }^{4}$ and also covalent amorphous semiconductors. ${ }^{5}$ Structural relaxation processes can for example be proven by a detection of a time dependent change of resistivity, ${ }^{6}$ of stress, ${ }^{7}$ or of mass density, ${ }^{8}$ by heat release, ${ }^{9}$ and more directly by positron annihilation spectroscopy, ${ }^{10}$ inelastic neutron scattering ${ }^{11}$ and spectroscopic studies. ${ }^{12}$ Advanced techniques such as molecular

\footnotetext{
${ }^{a}$ Author to whom all correspondence should be addressed.

b) Also at Laboratory for Neutron Scattering, ETH Zürich \& Paul Scherrer Institut, CH-5232 Villigen PSI, Switzerland.
}

dynamics ${ }^{13}$ and related methods ${ }^{14}$ allow for simulating this process theoretically. A parameter which is also strongly influenced by structural relaxation is the diffusivity of the atomic constituents. ${ }^{15}$ In general, a decrease of the selfdiffusivities is observed during annealing as a result of structural relaxation, depending, however, on the exact diffusion mechanism. The decrease of diffusivity is often correlated with a reduction and redistribution of defects in the asprepared material. This might be delocalized free volume as present in metallic glasses or localized point defects such as vacancies. During structural relaxation these defects may anneal out, a process which is generally accompanied by an increase in particle density in the order of $1 \% .{ }^{15}$ Keeping this in mind, a simple explanation for the decrease of diffusivities during relaxation can be found by a decrease in the concentration of defects governing diffusion. As a result, it can be stated that the determination of diffusivities during structural relaxation in combination with a measurement of the atomic density (or volume) allows one to identify the nature of this process. From basic considerations, structural relaxation, and defect annihilation are not necessarily related processes. It might also be possible that the amorphous structure is rearranged by a reorganization of bonds, bond angles, and a reduction of stress while the number of defects remain constant. $^{16}$

In the literature, structural relaxation based changes of the diffusivity were reported in so-called network glasses (silicates, germanates, borates) ${ }^{17}$ and also in metallic glasses. ${ }^{15}$ In covalently bound amorphous solids, such as, e.g., amorphous silicon, diffusion measurements were re- 
ported up to now only for fast transition metal impurity diffusers such as $\mathrm{Cu}$ or $\mathrm{Au} .{ }^{18}$ Here, diffusion takes place by a trap-limited mechanism and the change of diffusivities during annealing is attributed to the redistribution of traps and not of diffusion defects. No measurements were reported up to now for self-diffusion which takes place via diffusion defects. This might be due to the fact that diffusivity changes are insignificant for materials with a low glass transition temperature as speculated ${ }^{19}$ or that structural relaxation is not defect mediated. Another explanation might be that the experimental techniques commonly employed are too insensitive to detect a short time change of diffusivities due to structural relaxation in the amorphous state. From these considerations the following question arises: Is structural relaxation in amorphous covalent solids defect mediated, or not?

In order to clarify this open question, we carried out the present study to investigate the interrelation between selfdiffusivity changes and structural relaxation in a typical covalently bound amorphous semiconductor during isothermal annealing. To probe a possible time dependence of diffusivities we applied the method of neutron reflectometry on isotope multilayers. ${ }^{20}$ Neutron reflectivity has the advantage that it is isotope selective due to the fact that the nuclear potentials for neutrons vary strongly from isotope to isotope of the same element. It allows for the investigation of selfdiffusion in chemical homogeneous but isotope modulated layered materials.

In contrast to other methods of diffusivity determination (SIMS, radiotracer technique, NMR, QENS, etc.) neutron reflectometry is able to measure extremely low diffusivities below $10^{-23} \mathrm{~m}^{2} / \mathrm{s}$ and simultaneously self-diffusion lengths in the order of $1 \mathrm{~nm}$ and below. ${ }^{20}$ This is a prerequisite for a successful determination of structural relaxation related changes of diffusivity in covalent metastable materials. Low diffusivities occur due to covalent bonding, and short diffusion lengths have to be detected due to relatively short relaxation times in the region of interest and also due to the fact that crystallization has to be avoided. Using the already mentioned conventional methods for diffusion measurements, a possible diffusivity change as a function of annealing time is commonly averaged out due to the long annealing times which are necessary to measure diffusion lengths much larger than $1 \mathrm{~nm}$.

The most interesting and thoroughly investigated amorphous covalent system is amorphous silicon, which exhibits an ideal continuous random network. ${ }^{16}$ However, due to the fact that the neutron scattering lengths of the three stable silicon isotopes are quite similar $\left({ }^{28} \mathrm{Si}: 4.1 \mathrm{fm},{ }^{29} \mathrm{Si}: 4.7 \mathrm{fm}\right.$, and $\left.{ }^{30} \mathrm{Si}: 4.6 \mathrm{fm}\right)^{21}$ this system is not well suited for our experiments. Instead, we used near-stoichiometric silicon nitride $\left(\mathrm{SiN}_{x}, x=1.33\right)$ as a model system. Amorphous silicon nitride can be depicted as a random three-dimensional network consisting of silicon-centered nitrogen tetrahedra, which are joined at the corners. ${ }^{22}$ For neutron reflectometry experiments the material of interest has to be prepared in form of $\mathrm{Si}^{14} \mathrm{~N}_{x} / \mathrm{Si}^{15} \mathrm{~N}_{x}$ isotope multilayers, which are produced by magnetron sputtering of layers, alternating enriched with the two nitrogen isotopes. After deposition, a chemically homogeneous, but isotope-modulated silicon nitride layer is obtained. Due to the large difference in the coherent neutron scattering lengths of the two stable nitrogen isotopes ${ }^{14} \mathrm{~N}(9.37 \mathrm{fm})$ and ${ }^{15} \mathrm{~N}(6.44 \mathrm{fm}),{ }^{21}$ an ideal contrast for neutron scattering techniques is given. The isotope modulation manifests itself in Bragg peaks in the neutron reflectometry pattern. Due to isotope interdiffusion at elevated temperatures the one-dimensional order is destroyed and the Bragg peaks decrease and finally vanish. This effect can be used to determine nitrogen tracer diffusivities by an appropriate analysis. $^{20}$

\section{EXPERIMENTAL SETUP}

For the diffusion experiments isotope multilayers of the form $\mathrm{Si}^{14} \mathrm{~N}_{x} / \mathrm{Si}^{15} \mathrm{~N}_{x}$ were deposited by reactive r.f. magnetron sputtering. A mixture of 50 vol. $\%$ argon and 50 vol. $\%$ nitrogen, either nitrogen with natural isotope abundance $\left({ }^{14} \mathrm{~N}\right.$ concentration: $99.63 \%$ ) or isotopically enriched nitrogen $\left({ }^{15} \mathrm{~N}\right.$ concentration: $\left.99.5 \%\right)$ was used in combination with a silicon target. During deposition, alternating layers of $\mathrm{Si}^{14} \mathrm{~N}_{x}$ and $\mathrm{Si}^{15} \mathrm{~N}_{x}$ were formed. The whole multilayer consists of 6-20 bilayers with a thickness between 25 and $61 \mathrm{~nm}$. As substrates, Si wafers of dimension $20 \times 20 \times 2 \mathrm{~mm}^{3}$ were used. Sputter deposition was carried out with a planar magnetron source (AP\&T, Nürtingen, Germany). Deposition rates of $2-5 \mathrm{~nm} / \mathrm{min}$ were achieved, using an operating pressure of $5 \times 10^{-3}$ mbar and a sputtering power of $160 \mathrm{~W}$ at a substrate temperature of $400{ }^{\circ} \mathrm{C}$. The chemical composition of the sputtered layers was measured by non-Rutherford backscattering spectroscopy to be near-stoichiometric $\mathrm{SiN}_{x}$ $(x=1.33){ }^{23}$ The $\mathrm{O}$ impurity concentration was determined to be less than 0.2 at. $\%$, and the hydrogen content was measured to be about 0.2 at. \% by additional nuclear reaction analysis. ${ }^{23}$ Structural characterization of the layers was carried out by $\mathrm{x}$-ray diffractometry and Fourier transform infrared spectrometry both confirming the amorphous state of the material. Diffusion annealing was carried out between 900 and $1350{ }^{\circ} \mathrm{C}$ in nitrogen atmosphere at ambient pressure by placing two samples with the surfaces on each other in a silicon nitride powder pack.

The diffusion experiments were carried out by neutron reflectometry using the reflectometers AMOR and MORPHEUS at the Swiss spallation neutron source (SINQ), located at the Paul Scherrer Institute (Switzerland). The first reflectometer works in the time-of-flight mode at incoming neutron wavelengths between 0.2 and $0.9 \mathrm{~nm}$. Reflectivity patterns were measured at different incident angles between $0.3^{\circ}$ and $1.8^{\circ}$. The second reflectometer was operated in angle dispersive $(\theta / 2 \theta)$ mode using a monochromatic neutron beam at a wavelength of $0.474 \mathrm{~nm}$.

\section{RESULTS}

In Fig. 1 the neutron reflectivity pattern of an asdeposited $\left[\mathrm{Si}^{14} \mathrm{~N}_{x}(19 \mathrm{~nm}) / \mathrm{Si}^{15} \mathrm{~N}_{x}(19 \mathrm{~nm})\right]_{12}$ multilayer is shown. Bragg peaks of first, third, fifth, and seventh order occur at scattering wave vectors of $q_{z}=4 \pi \sin \theta / \lambda$ $=0.024 \AA^{-1}, 0.053 \AA^{-1}, 0.085 \AA^{-1}$, and $0.117 \AA^{-1}$ due to the periodic variation of the neutron scattering length of the 


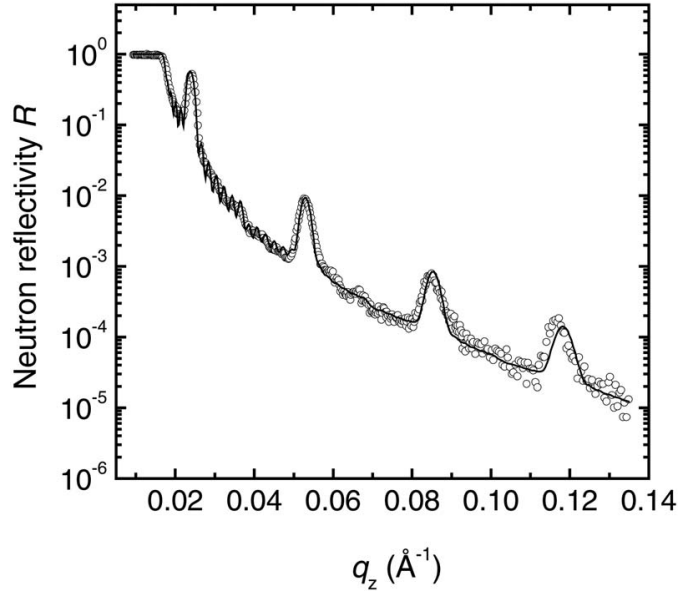

FIG. 1. Neutron reflectivity pattern of a an as-deposited $\left[\mathrm{Si}^{14} \mathrm{~N}_{x}(19 \mathrm{~nm}) / \mathrm{Si}^{15} \mathrm{~N}_{x}(19 \mathrm{~nm})\right]_{12}$ isotope multiplayer. The line corresponds to a fit with the program PARRATT $32^{34}$.

two isotopes. As demonstrated by secondary neutral mass spectrometry, ${ }^{24}$ the $\mathrm{Si}$ and overall $\mathrm{N}$ concentration remains constant with depth showing the chemical homogeneity of the stack layer, while there exists an isotopic modulation of nitrogen. Also shown in Fig. 1 is a least-squares fit of the neutron reflectivity pattern by a computer program based on Parratt's formalism. ${ }^{25}$ The bilayer thickness was determined to be $(38.2 \pm 0.3) \mathrm{nm}$. The diffusion experiments were carried out on this (or similar) isotope multilayers with a bilayer thickness between 25 and $61 \mathrm{~nm}$.

In Fig. 2 the first order Bragg peak for a multilayers of type $\left[\mathrm{Si}^{14} \mathrm{~N}_{x}(21 \mathrm{~nm}) / \mathrm{Si}^{15} \mathrm{~N}_{x}(7 \mathrm{~nm})\right]_{20}$ is shown for samples in the as-deposited state and after diffusion annealing at $1150{ }^{\circ} \mathrm{C}$ for different times between 15 and $5400 \mathrm{~min}$. The reflectivity is multiplied by $q_{z}^{4}$ in order to correct for the Fresnel reflectivity of the (idealized) substrate. An intensity decrease for the Bragg peak as a function of annealing time

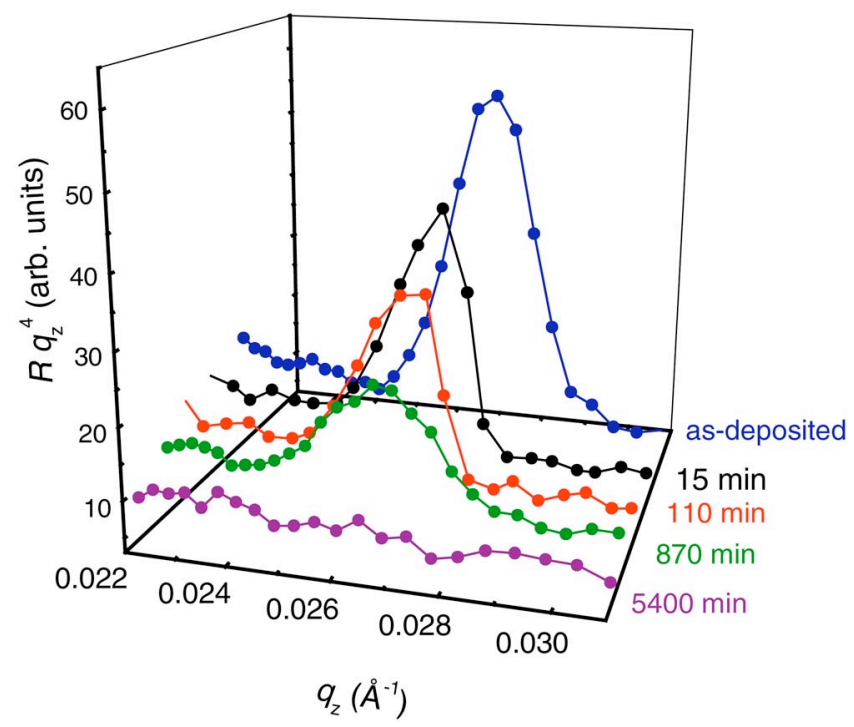

FIG. 2. (Color online) Neutron reflectivity patterns of a $\left[\mathrm{Si}^{14} \mathrm{~N}_{x}(21 \mathrm{~nm}) / \mathrm{Si}^{15} \mathrm{~N}_{x}(7 \mathrm{~nm})\right]_{20}$ isotope multilayer in the as-deposited state and after annealing for various times at $1150{ }^{\circ} \mathrm{C}$ in the vicinity of the first order Bragg peak. The reflectivity is multiplied by $q_{z}^{4}$ in order to correct for the Fresnel reflectivity of the (idealized) substrate.

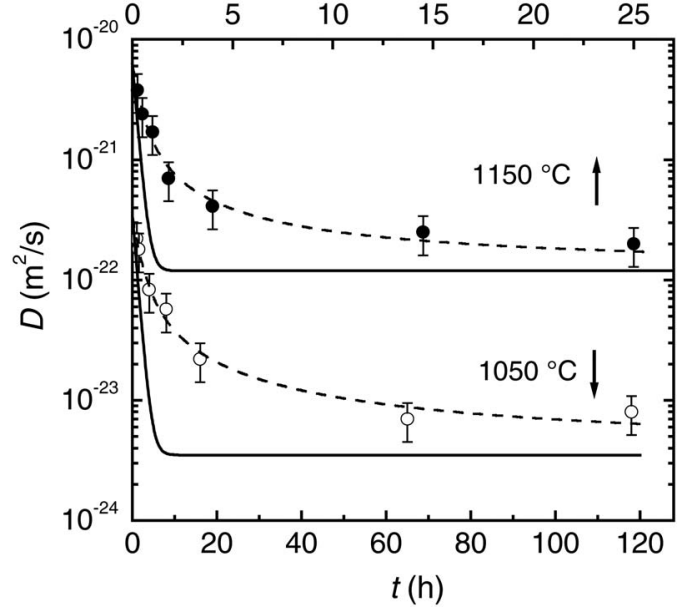

FIG. 3. Average self-diffusivities, $D_{\mathrm{av}}$, of nitrogen in amorphous silicon nitride films for various annealing times at 1050 and $1150{ }^{\circ} \mathrm{C}$ (dots). The dashed line corresponds to a fit of Eq. (5) to the experimental data and the solid line to the instantaneous diffusivities, $D$, as calculated from this fit according to Eq. (4).

is clearly visible for the annealed samples, increasing with annealing time. The self-diffusivities can be calculated according to the expression ${ }^{26}$

$$
I(t)=I_{0} \exp \left(-\frac{8 \pi^{2} n^{2} D}{d^{2}} t\right),
$$

where $I_{0}$ is the intensity of the $n$th order Bragg peak at time $t=0, D$ is the diffusivity, and $d$ is the bilayer thickness. The diffusivities as a function of annealing time for measurements at 1050 and $1150{ }^{\circ} \mathrm{C}$ are given in Fig. 3. Obviously, the diffusivity depends on time. The diffusivities decrease by about more than one order of magnitude with increasing annealing time. This time dependence of the diffusivities can be interpreted to be the result of structural relaxation of the amorphous network structure as explained in the Introduction. Point defects responsible for diffusion are annihilated at sinks during annealing.

The diffusivities shown in Fig. 3 are time-averaged diffusivities, $D_{\text {av }}$, which are given by

$$
D_{\mathrm{av}}(t)=\frac{1}{t} \int_{0}^{t} D\left(t^{\prime}\right) d t^{\prime},
$$

where $D$ is the instantaneous diffusivity. ${ }^{15}$ Differentiating Eq. (2) we get

$$
D(t)=D_{\mathrm{av}}(t)+t \frac{\partial D_{\mathrm{av}}(t)}{\partial t} .
$$

This expression can be used to calculate $D$ from the time dependence of $D_{\mathrm{av}}$. The data together with fit curves are given in Fig. 3. The main result is that after a sharp drop $D(t)$ becomes constant in time. This means that a relaxed amorphous state is reached, where further annealing does not alter diffusivities. The diffusivity in the relaxed amorphous state is termed $D_{R}$ and corresponds approximately to $D_{\mathrm{av}, R}$ for long annealing times. For structural relaxation processes, the decrease of the instantaneous diffusivity can be described as ${ }^{15}$ 


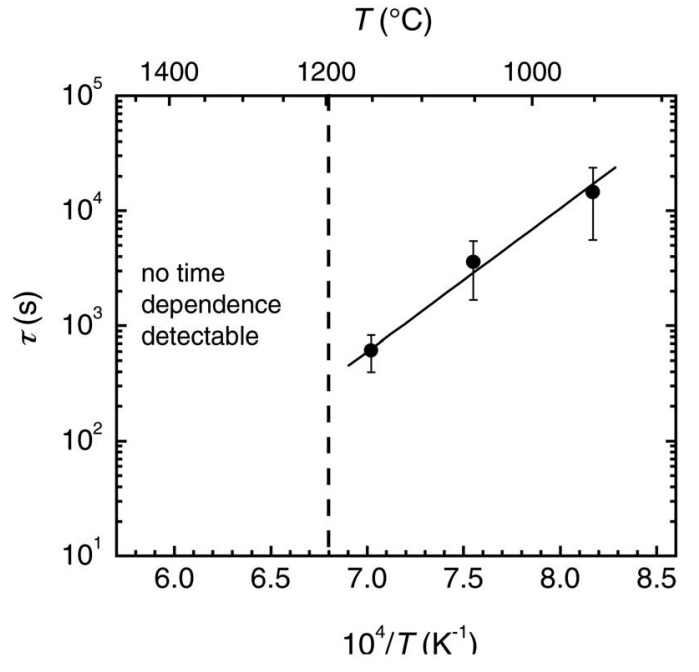

FIG. 4. Time constant of relaxation, $\tau$, for the nitrogen diffusivities in amorphous silicon nitride films as a function of reciprocal temperature.

$$
D(t)=D_{R}+\left(D_{0}-D_{R}\right) \exp \left(-\frac{t}{\tau}\right)
$$

with $D_{0}$ as the (unknown) value at $t=0$ and $\tau$ as a typical relaxation time. Introducing Eq. (4) in Eq. (2) we get

$$
D_{a v}(t)=D_{R}+\left(D_{0}-D_{R}\right) \frac{\tau}{t}\left(1-\exp \left(-\frac{t}{\tau}\right)\right) \text {. }
$$

This equation is used by least-squares fitting the experimental data in Fig. 3 in order to determine $D_{R}$ and $\tau$. Analogous experiments as for $1150{ }^{\circ} \mathrm{C}$ and $1050{ }^{\circ} \mathrm{C}$ were carried out at temperatures of $950{ }^{\circ} \mathrm{C}, 1250{ }^{\circ} \mathrm{C}$, and $1350{ }^{\circ} \mathrm{C}$, where silicon nitride is in the amorphous state for the annealing times used. ${ }^{23}$ For temperatures of $1150{ }^{\circ} \mathrm{C}$ and below a decrease of the diffusivity due to structural relaxation is observed. In contrast, at higher temperatures this effect is experimentally not detectable, probably due to the fact that the relaxation time $\tau$ is too short. After an annealing time of $10 \mathrm{~min}$, which is the shortest time that is possible with our experimental facility, all defects are annihilated and the diffusivity of the relaxed state is measured.

The $\tau$ values determined by the fitting procedure are shown in Fig. 4 as a function of reciprocal temperature. In first approximation the data obey the Arrhenius law according to

$$
\tau=\tau_{0} \exp \left(\Delta H_{\tau} / k_{B} T\right)
$$

and the activation enthalpy can be assessed to $\Delta H_{\tau}$ $=\left(2.6_{-1.0}^{+0.6}\right) \mathrm{eV}$. The rather large errors are due to the limited number of only three data points which can be used for analysis.

It is interesting to note that within the temperature range between 1150 and $1250{ }^{\circ} \mathrm{C}$, where the time dependence of the diffusivity vanishes, the relaxation time reaches a value of $\tau=100 \mathrm{~s}$ as can be deduced by extrapolating from lower temperatures. This is a characteristic time by which commonly the glass transition temperature of an amorphous system is defined ${ }^{1}$ and the transition from a nonequilibrium frozen-in amorphous state to a metastable state is observed.

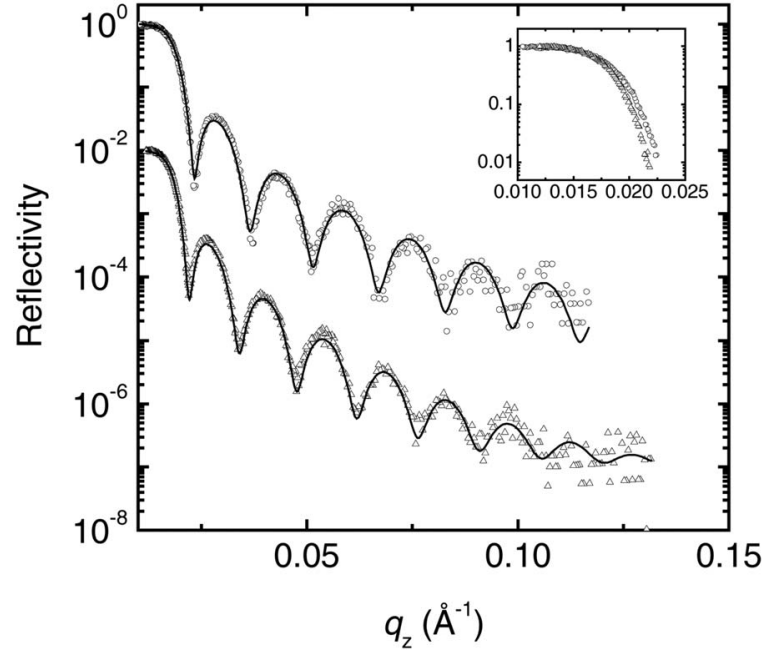

FIG. 5. Neutron reflectivity pattern of a an as-deposited $\left[\mathrm{Si}^{14} \mathrm{~N}_{x}(19.4 \mathrm{~nm}) / \mathrm{Si}^{15} \mathrm{~N}_{x}(19.4 \mathrm{~nm})\right]$ layer $(\bigcirc)$ and the same layer annealed for $14 \mathrm{~h}$ at $1150{ }^{\circ} \mathrm{C}(\triangle)$. The lines correspond to a fit with the program PARRATT32. ${ }^{34}$ The inset shows the enlarged data in the vicinity of the reflection edge.

At this point (or better: Range) the structure and also the defect concentration reaches a metastable equilibrium and the system can rearrange itself on the time scale of experimental observation. Eventually, our data are an indication for such a transition. Otherwise, it is still unclear at the moment whether amorphous silicon nitride can be considered as a true glass-forming material at all, since no corresponding liquid phase does exist and the solid decomposes at a high temperature of about $1880{ }^{\circ} \mathrm{C}$ at 1 bar. $^{27}$

The present experiments with neutron reflectometry also exhibited that during diffusion annealing not only a decrease in the intensity of the Bragg peaks occurs due to isotope interdiffusion but also a shift of the Bragg peaks and a shift of the reflectivity edge at $0.017 \AA^{-1}$ (see Fig. 1) to lower $q_{z}$ values. Qualitatively, this can be explained with an increase in the layer thickness and a simultaneous decrease in the scattering density, which means that a decrease in particle density due to an increase of the film volume takes place if the number of scatterers is constant. For a closer, more quantitative inspection of that phenomenon a special experiment was designed. A single amorphous $\mathrm{Si}^{15} \mathrm{~N}_{x} / \mathrm{Si}^{14} \mathrm{~N}_{x}$ bilayer with a thickness of about $38 \mathrm{~nm}$ was sputtered on a silicon substrate and annealed at $1150{ }^{\circ} \mathrm{C}$ for $14 \mathrm{~h}$ in nitrogen atmosphere in analogy to the diffusion experiment which gave the diffusivity in the relaxed state (see Fig. 3). The results of this experiment are shown in Fig. 5. In contrast to Fig. 1 no Bragg peaks occur but fringes resulting from reflection and interference of the neutrons at the silicon/silicon nitride interface and the air/silicon nitride interface. The isotope contrast of the single $\mathrm{Si}^{15} \mathrm{~N}_{x} / \mathrm{Si}^{14} \mathrm{~N}_{x}$ layer is too small to be detected with the experimental equipment used. In the asdeposited state the reflectivity can be fitted using a layer thickness of $d=38.9 \mathrm{~nm}$ and a scattering density of $\rho_{s}$ $=5.60 \times 10^{-6} \AA^{-2}$. The scattering density is the average of the scattering density of both isotopes of $4.78 \times 10^{-6} \AA^{-2}$ and $6.42 \times 10^{-6} \AA^{-2}$, respectively. In contrast, fitting of the annealed sample gives a higher layer thickness of $41.8 \mathrm{~nm}$ 


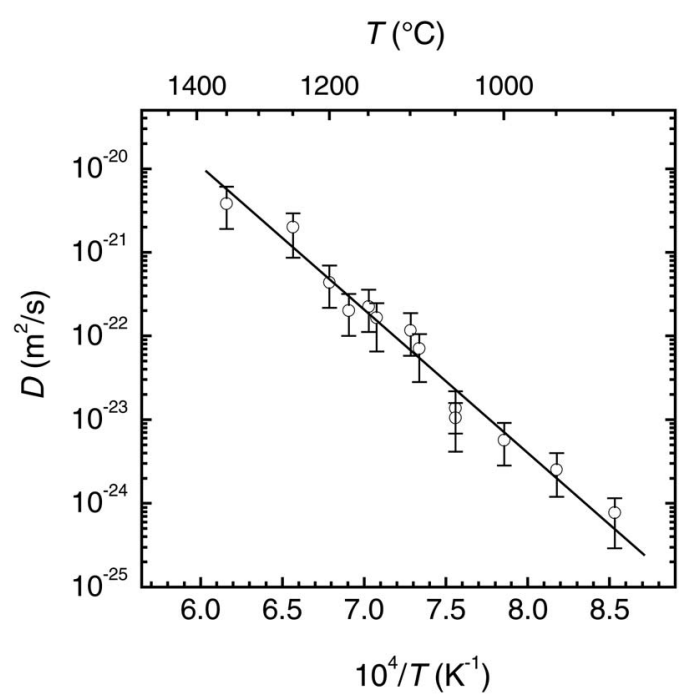

FIG. 6. Nitrogen diffusivities in relaxed amorphous silicon nitride films as a function of reciprocal temperature. The diffusivities between 950 and $1250{ }^{\circ} \mathrm{C}$ were taken from Ref. 20.

and a lower scattering density of $5.30 \times 10^{-6} \AA^{-2}$. Assuming the number of scattering atoms, $\mathrm{N}$, to be constant, the change in particle density can be calculated independently from (1) $\Delta \rho_{p} / \rho_{p}=\Delta \rho_{s} / \rho_{s}=(5.4 \pm 2.4) \%$ with $\rho_{p}=\rho_{s} /\left(4 b_{\mathrm{N}}+3 b_{\mathrm{Si}}\right)$ and (2) $\Delta \rho_{p} / \rho_{p}$ $\approx-\Delta d / d=(7.4 \pm 0.7) \%$ with $\rho_{p}=N / V=N /\left(d L^{2}\right)\left(L^{2}:\right.$ Sample surface, $b_{i}$ : Neutron scattering length of element $i$ ). Both values are identical within error limits, which means that during structural relaxation a density decrease caused by a volume increase occurs. Analysis of similar experiments at other temperatures gave the same result within error limits.

For further analysis the nitrogen self-diffusivities in the relaxed state, $D_{\mathrm{av}, R}$ are plotted in Fig. 6 as a function of reciprocal temperature between 900 and $1350{ }^{\circ} \mathrm{C}$. Here data from a preliminary study ${ }^{20}$ are given and new additional diffusivities from the present experiments are also added.

A least-squares fit of the experimental data to the Arrhenius equation

$$
D=D_{0} \exp \left(-\Delta H_{D} / k_{B} T\right)
$$

results in an activation enthalpy of diffusion of $\Delta H_{D}$ $=(3.4 \pm 0.3) \mathrm{eV}$ and in a corresponding preexponential factor of $D_{0}=2 \times 10^{-10} \mathrm{~m}^{2} / \mathrm{s}$ (error: $\log D_{0}= \pm 1.0$ ).

\section{DISCUSSION}

The results presented in the last section show that during isothermal annealing at temperatures between 950 and $1150{ }^{\circ} \mathrm{C}$ structural relaxation takes place, which has a significant influence on the nitrogen diffusivities. A decrease of the diffusivities is observed as a function of the ongoing relaxation process until a relaxed state is reached. At the same time a density decrease is observed on heating the amorphous silicon nitride layers. These results demonstrate that structural relaxation is caused by a defect annihilation mechanism. During annealing the number of defects governing diffusion is reduced by more than one order of magnitude, which will result in a rearrangement of the amorphous network and its structural properties. The observation that the density of amorphous silicon nitride is decreasing during relaxation distinguishes this material from other types of amorphous materials, such as metallic glasses, ${ }^{15}$ silica glasses, ${ }^{2}$ and also amorphous silicon carbide, ${ }^{5}$ which all show densification during annealing. The only material known to the authors, which shows an abnormal density change during structural relaxation, is amorphous silicon, where the density remains approximately constant within $0.1 \%{ }^{28}$ More advanced measurements show that the density varies in a nonlinear way within this range during nonisothermal heating from room temperature to $250{ }^{\circ} \mathrm{C}$. The present measurements prove that structural relaxation during isothermal annealing is not necessarily accompanied by a densification of the amorphous solid and that also a density decrease as high as $5 \%$ can occur.

From our results closer information on the nature of the annihilation process can be derived. Structural relaxation in metallic glasses is often described by the free volume concept, ${ }^{29}$ which is very successful for random close-packed solids. The amount of free volume is reduced during relaxation by a rearrangement of atoms, which leads to an increase in density and a decrease in diffusivities. In the present case of covalently bound solids the concept of point defects is more appropriate to describe the situation. In general, the natural defects in amorphous semiconductors are dangling or floating bonds. ${ }^{30}$ However, vacancies and interstitial are also assumed to be stable. ${ }^{31,32}$ Now, the simplest way to explain the observed relaxation process is a preferential annihilation of interstitial-like defects or defect clusters during heating. A decrease in the number of interstitials leads to a decrease in atomic density and to an increase in volume. A simultaneous decrease of the interstitial concentration and of the diffusivities implies that during structural relaxation nitrogen self-diffusion is also governed by an indirect interstitial mechanism (interstitialcy mechanism), where the diffusivity is proportional to the interstitial concentration. These results also imply that during sputtering a large amount of nonthermal interstitials have to be formed, which are annihilated during annealing.

In the relaxed state the temperature dependence of diffusivity is given by Eq. (7). Where preliminary results of this work were published, ${ }^{20}$ it is argued by comparison to diffusivities measured in crystalline silicon nitride ${ }^{33}$ that in relaxed amorphous silicon nitride a direct diffusion mechanism via structural defects is acting without the mediation of thermal point defects. In that case the activation enthalpy of diffusion is essentially determined by the defect migration enthalpy $\left(\Delta H_{D}=\Delta H_{m}\right)$. It can now be argued that also in the relaxed amorphous state structural interstitials are still present, which are not completely annihilated by structural relaxation. From the measurements given in Fig. 3 we can assess the maximum decrease of the instantaneous diffusivity during relaxation to be roughly a factor of 100 . It can now be assumed that the concentration of interstitials, $c_{I}$, decreases by the same amount, if $D \sim c_{I}$. We further assume that the annihilation of an interstitial at a free surface increases the volume by $\Omega$ (one atomic volume) and we obtain $\Delta V / V$ $=\left(I_{i}-I_{e}\right) \Omega /(N \Omega)=\left(I_{i}-I_{e}\right) / N \approx 0.05$, where $I_{i}$ is the initial and $I_{e}$ the final number of interstitials and $N$ is the number of 
atoms. Using further $I_{e}=0.01 I_{i}$ we receive $c_{I, R}=I_{e} / N \approx 5$ $\times 10^{-4}$ for the interstitial concentration after the structural relaxation process. The diffusivity in the relaxed state is now given by

$$
\begin{aligned}
D_{R} & =D_{0} \exp \left(-\Delta H_{m} / k_{B} T\right) \\
& =c_{I, R} a^{2} \nu \exp \left(\Delta S / k_{B}\right) \exp \left(-\Delta H_{m} / k_{B} T\right),
\end{aligned}
$$

where $a=0.26 \mathrm{~nm}$ is the jump length, $\nu=10^{13} \mathrm{~s}^{-1}$ is the Debye frequency, and $\Delta S$ is the entropy of diffusion. Using the results in Fig. 6, $\Delta S$ can be calculated to be about $0-2 k_{B}$, which is quite reasonable. If we use instead the expression $D_{0}=a^{2} \nu \exp \left(\Delta S / k_{B}\right)$ for the preexponential factor, as valid for a diffusion mechanism with thermal point defects, we obtain a unrealistically large negative value of $\Delta S=-8 k_{B}$. From this we can conclude that also in the relaxed state diffusion is very likely mediated via structural interstitials. Further support of this assumption might be found in looking at the activation enthalpy of relaxation, $\Delta H_{\tau}$, which ranges between 1.6 and $3.3 \mathrm{eV}$. This quantity has to be associated with the activation enthalpy of defect motion during defect annihilation. Consequently $\Delta H_{\tau} \approx \Delta H_{m}$ has to be valid if the same diffusion mechanism is acting in the relaxed and unrelaxed state. From the present data this is difficult to decide. Within error limits the upper condition is indeed fulfilled. Nevertheless, the present results also allow an interpretation where $\Delta H_{\tau}<\Delta H_{m}$. For that case atomic motion in the relaxed state has to be governed by an energetically less favorable mechanism than that acting during structural relaxation. This means that other defects than interstitials with a higher concentration (e.g., vacancies) might be involved. Such a mechanism for relaxed amorphous silicon nitride cannot be ruled out completely on basis of the present results.

\section{CONCLUSION}

We carried out nitrogen diffusion studies on amorphous silicon nitride films as a function of annealing time at constant temperature. The results revealed a decrease of the nitrogen diffusivity with increasing annealing time at temperatures between 950 and $1150{ }^{\circ} \mathrm{C}$ and a simultaneous decrease of the film density. This behavior is interpreted as structural relaxation of the amorphous network structure, which is caused by an annihilation of interstitial-like defects. From a comparison of diffusivities in relaxed and unrelaxed amorphous silicon nitride we conclude that very likely the same diffusion mechanism is acting in both states.

\section{ACKNOWLEDGMENTS}

We dedicate this work to Professor Dr. Günter Borchardt on the occasion of his 65th birthday. This work is based on experiments performed at the Swiss Spallation Neutron
Source SINQ, and the Paul Scherrer Institute, Villigen, Switzerland. This research project has been supported by the European Commission under the 6th Framework Programme through the Key Action: Strengthening the European Research Area, Research Infrastructures, Contract No. RII3-CT2003-505925. Financial support by the German Research Foundation (DFG) is gratefully acknowledged.

${ }^{1}$ C. A. Angell, K. L. Ngai, G. B. McKenna, P. F. McMillan, and S. W. Martin, J. Appl. Phys. 88, 3113 (2000).

${ }^{2}$ U. Buchenau, H. M. Zhou, N. Nucker, K. S. Gilroy, and W. A. Phillips, Phys. Rev. Lett. 60, 1318 (1988).

${ }^{3}$ J. M. Hutchinson, Prog. Polym. Sci. 20, 703 (1995).

${ }^{4}$ A. van den Beukel and S. Radelaar, Acta Metall. 31, 419 (1983).

${ }^{5}$ M. Ishimaru, I.-T. Bae, Y. Hirotsu, S. Matsumura, and K. E. Sickafus, Phys. Rev. Lett. 89, 055502 (2002).

${ }^{6}$ S. G. Mayr, J. Appl. Phys. 97, 096103 (2005).

${ }^{7}$ C. A. Volkert, J. Appl. Phys. 74, 7107 (1993).

${ }^{8}$ K. Russew and F. Sommer, J. Non-Cryst. Solids 319, 289 (2003).

${ }^{9}$ S. Roorda, S. Doorn, W. C. Sinke, P. M. L. O. Scholte, and E. van Loenen, Phys. Rev. Lett. 62, 1880 (1989).

${ }^{10}$ C. Nagel, K. Rätzke, E. Schmidtke, F. Faupel, and W. Ulfert, Phys. Rev. B 60, 9212 (1999).

${ }^{11}$ A. Meyer, H. Schober, and D. B. Dingwell, Europhys. Lett. 59, 708 (2002).

${ }^{12}$ C. Demoulin, C. J. Montrose, and N. Ostrowsk, Phys. Rev. A 9, 1740 (1974).

${ }^{13}$ I. Stich, R. Car, and M. Parrinello, Phys. Rev. B 44, 11092 (1991).

${ }^{14}$ G. T. Barkema and N. Mousseau, Phys. Rev. Lett. 81, 1865 (1998).

${ }^{15}$ F. Faupel, W. Frank, M. P. Macht, H. Mehrer, V. Naundorf, K. Rätzke, H. R. Schober, S. K. Sharma, and H. Teichler, Rev. Mod. Phys. 75, 237 (2003).

${ }^{16}$ S. Roorda, W. C. Sinke, J. M. Poate, D. C. Jacobson, S. Dierker, B. S. Dennis, D. J. Eaglesham, F. Spaepen, and P. Fuoss, Phys. Rev. B 44, 3702 (1991).

${ }^{17}$ U. Schoo and H. Mehrer, Solid State Ionics 130, 243 (2000).

${ }^{18}$ S. Coffa, J. M. Poate, D. C. Jacobson, W. Frank, and W. Gustin, Phys. Rev. B 45, 8355 (1992).

${ }^{19}$ K. Hoshino, R. S. Averback, H. Hahn, and S. J. Rothman, Defect Diffus. Forum 59, 225 (1988).

${ }^{20}$ H. Schmidt, M. Gupta, and M. Bruns, Phys. Rev. Lett. 96, 055901 (2006).

${ }^{21}$ V. F. Sears, Neutron News 3, 26 (1992).

${ }^{22}$ J. F. Justo and C. R. S. Silva, Defect Diffus. Forum 206, 19 (2002).

${ }^{23}$ H. Schmidt, W. Gruber, G. Borchardt, M. Bruns, M. Rudolphi, and H. Baumann, Thin Solid Films 450, 344 (2004).

${ }^{24}$ H. Schmidt, M. Gupta, U. Geckle, and M. Bruns, Defect Diffus. Forum 263, 51 (2007).

${ }^{25}$ L. G. Parratt, Phys. Rev. 95, 359 (1954).

${ }^{26}$ J. Speakman, P. Rose, J. A. Hunt, N. Cowlam, R. E. Somekh, and A. L. Greer, J. Magn. Magn. Mater. 156, 411 (1996).

${ }^{27}$ F. L. Riley, J. Am. Ceram. Soc. 83, 245 (2000).

${ }^{28}$ J. A. Roth, G. L. Olsen, D. C. Jacobson, and J. M. Poate, Appl. Phys. Lett. 57, 1340 (1990).

${ }^{29}$ F. Spaepen, Acta Metall. 25, 407 (1976).

${ }^{30}$ S. T. Pantelides, Phys. Rev. Lett. 57, 2979 (1986).

${ }^{31}$ G. N. van den Hoven, Z. N. Liang, L. Niesen, and J. S. Custer, Phys. Rev. Lett. 68, 3714 (1992).

${ }^{32}$ C. R. Miranda, A. Antonelli, A. J. R. da Silva, and A. Fazzio, J. NonCryst. Solids 338-340, 400 (2004).

${ }^{33}$ H. Schmidt, U. Geckle, and M. Bruns, Phys. Rev. B 74, 045203 (2006).

${ }^{34}$ C. Braun, Parratt32 or the reflectometry tool, HMI, Berlin (19971999); http://www.hmi.de/bensc/instrumentation/instrumente/v6/refl/ parratt_en.htm. 